\title{
Picture-to-Amount (PITA): Predicting Relative Ingredient Amounts from Food Images
}

\author{
Jiatong $\mathrm{Li}^{*}$, Fangda Han*, Ricardo Guerrero ${ }^{\dagger}$ and Vladimir Pavlovic*† \\ ${ }^{*}$ Department of Computer Science, Rutgers University, Piscataway, NJ, USA \\ ${ }^{\dagger}$ Samsung AI Center, Cambridge, UK
}

\begin{abstract}
Increased awareness of the impact of food consumption on health and lifestyle today has given rise to novel data-driven food analysis systems. Although these systems may recognize the ingredients, a detailed analysis of their amounts in the meal, which is paramount for estimating the correct nutrition, is usually ignored. In this paper, we study the novel and challenging problem of predicting the relative amount of each ingredient from a food image. We propose PITA, the Picture-toAmount deep learning architecture to solve the problem. More specifically, we predict the ingredient amounts using a domaindriven Wasserstein loss from image-to-recipe cross-modal embeddings learned to align the two views of food data. Experiments on a dataset of recipes collected from the Internet show the model generates promising results and improves the baselines on this challenging task. A demo of our system and our data is available at: foodai.cs.rutgers.edu.
\end{abstract}

\section{INTRODUCTION}

Increased awareness of the impact of food consumption on health and lifestyle today has given rise to novel datadriven food analysis systems, e.g., [1], [2], whose goals are to find effective and efficient ways for tracking daily food intake and, subsequently, enable automatic dietary assessment or even facilitate possible positive changes in lifestyle. Many of those systems use data modalities such as images to seamlessly extract information related to the food item that was consumed, where the information includes the identity of the meal, its ingredients, or even its caloric value. While these systems frequently claim to predict the energy intake, they base these predictions on standard energy tables of standardized ingredients (e.g., USDA ${ }^{1}$ ). The estimation of the food amount, a highly challenging and often ambiguous task, is delegated to the users themselves. Even systems that aim to predict a finegrained ingredient-based representation of the food item, e.g., [3], [4], do not consider the problem of predicting the ingredient amounts or relative contributions of ingredients in each dish. However, these amounts are paramount for estimating the correct energy value of the meal. A small amount of highfat food might not be a major health risk factor, while an unhealthy ingredient with a dominant amount may lead to potential health problems. Analyzing individual nutrients of a food dish in detail and how each ingredient contributes to health through its nutritions can resolve the immediate and important goal of dietary assessment. Estimating the finegrained ingredient information can also facilitate choices of which ingredients could be replaced according to users' dietary

\footnotetext{
${ }^{1}$ https://ndb.nal.usda.gov/ndb/search/list
}

needs, leading to overall improvements in nutritional behavior. Therefore, we argue there is a need for food analysis systems which output fine-grained ingredient amounts.

In this paper, we study the novel problem of predicting the relative amount of each ingredient in a food item from images, where relative amounts are the proportions of each ingredient needed to prepare the food, relative to the total ingredient weight. Scaling all ingredient amounts to the same factor will not change the food or recipe itself. Only the number of servings will be changed.

Several critical challenges are associated with ingredient amount predictions. First, some ingredients, like salt, are invisible. The existence and amounts of these ingredients can only be inferred through correlation with other ingredients. However, these ingredients cannot be simply ignored in food analysis systems. They are important in the cooking process as they impart specific flavors to the dish, while at the same time they can be crucial in estimating nutritional values, c.f., [5]. Therefore, it is essential to consider invisible ingredients. Second, cooking involves complex operations that transform ingredients both mechanically and chemically. Attributes such as shape and color can change drastically during this process. Last, the ingredients are very diverse and distributed extremely unevenly in different dishes, leading to high-dimensional and highly unbalanced estimation problems.

To tackle these challenges, we propose the PITA framework. More specifically, we aim at first learning an embedding representation of the image from a cross-modal image-totext retrieval system, such as [6]. When learning the retrieval model, ingredient co-occurrences and cooking actions are encoded by the text encoder. This information is related to the image appearance through the image encoder and the shared image-text features. As illustrated in Figure 1, embeddings are used to condition ID and, in a cascade, AP. In this manner, the model drives the cross-modal embedding to be highly relevant to the task of estimation, while being recursively conditioned from simpler (detection) to more complex tasks (estimation). The unbalanced problem can be addressed by the positive sample weight in the ID loss. During estimation, the relationship of amounts are learned by AP using Wasserstein distance, the transport loss which ideally matches our problem because ingredients can be classified into functional groups like spices and oil. The penalty of predicting vegetable oil as olive oil should be less than that of predicting meat as vegetables. The ingredient functional substitution groups are 


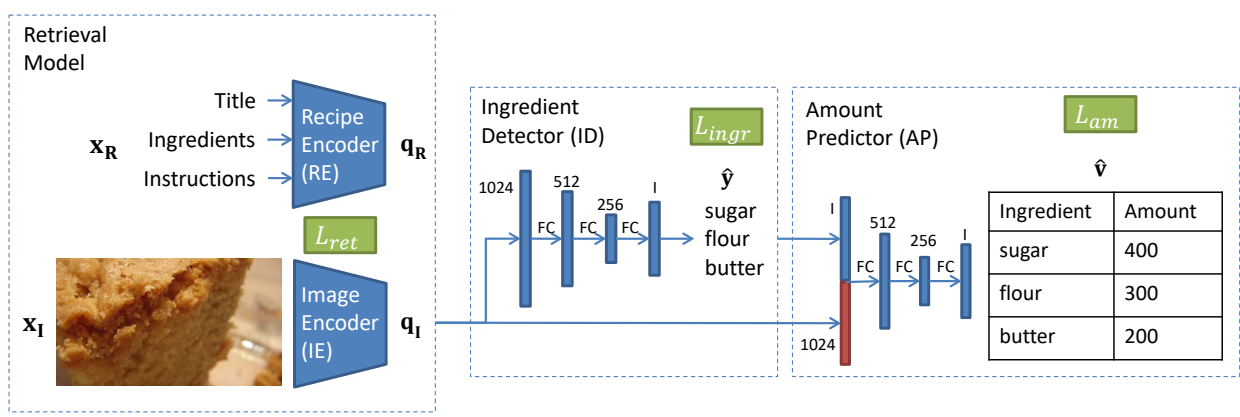

Fig. 1: Overview of our PITA approach. We first learn an embedding representation from retrieval system. We proceed to detect the ingredients from embeddings and finally predict amounts from embeddings and detected ingredients.

first selected by Word2vec-like [7] cosine similarities which are trained on titles, ingredients and instructions of Recipe1M [3]. Ingredient pairs above a certain threshold are proposed, then curated by human annotators. The distance matrix of the Wassterstein transport is determined by the substitution groups and the cosine distances of Word2vec embeddings between ingredients. When constructing the functional groups, the dimensions are also reduced. Canonical ingredient construction reduces the number of total ingredients (output dimension) from $16 \mathrm{k}$ in Recipe $1 \mathrm{M}$ to $1.4 \mathrm{k}$. The ingredients form 172 substitution groups, which further reduces the complexity and enhances generalization of the approach. Experiments on a dataset of recipes collected from the Internet show the model generates state-of-the-art results, improving previous baselines.

In summary, the contributions of this paper are:

- We propose a novel and challenging problem: analyzing the relative amounts of the ingredients from a food image.

- We define a novel semi-automatic process to create ingredient substitution groups and facilitate data-driven functional ingredient equivalencies.

- We propose PITA deep learning framework to solve the problem of relative ingredient-specific amount estimation and improve previous baselines.

\section{RELATED WORK}

A growing body of research in computer vision has focused on the problems of computational food classification, crossmodal retrieval, and ingredient analysis. While prior work has addressed those difficult problems, more demanding tasks of food volume and food amount estimation, needed for detailed nutritional analysis, are emerging as the key topics in computational food analysis and challenges for computer vision and machine learning communities.

Food Classification. Hand-crafted features and traditional classifiers were initially used to classify food images into food categories or meal types, such as vegetables, soups, fish, grilled fish, etc. Both [1] and [8] used SVMs as classifier. [8] leveraged super-pixels in combination with random forests, while [1] employed a bag-of-visual-words approach. [1] also estimated the energy caloric content of a meal from a restaurant menu based on the classification results. Classical computer vision features tend to be outperformed by modern methods that rely on deep learning for feature extraction or directly using deep learning end-to-end. [9] and [10] used deep learning features while [11], [12], [13], [14] and [15] trained deep-learning models for food recognition. [16] introduced slice convolution blocks and [17] adversarially mines discriminative food regions for food classification.

Cross-modal Retrieval. In the context of the work proposed here, this means that given an image, the system retrieves its recipe from a collection of test recipes or vice-versa. [18] proposed a framework that learns a joint embedding of recipe ingredients and images while [3] leveraged titles, ingredients and instructions of recipes. In AdaMine [19], recipes from the same semantic class were embedded closer. [20] incorporated attention mechanism to the recipe embedding in order to focus on words that facilitate retrieval. ACME [21] imposed modality alignment and cross-modal translation consistency using an adversarial learning strategy to improve the retrieval performance. Although cross-modal retrieval can be applied to the ingredient amount estimation problem by returning the ingredients and amounts of the best matching recipe in the database of recipes, this approach cannot readily generalize to novel foods and highly depends on the collection of recipes.

Ingredient Analysis. A significantly more challenging task is predicting the existence of ingredients given a food image. [4] used multi-task deep learning and a graph modeling ingredient co-occurrences. [22] predicted ingredients as sets and generated cooking instructions by attending to both image and its inferred ingredients simultaneously. [23] developed an ingredient-guided cascaded multi-attention network for ingredient detection. However, these works do not tackle the quantity prediction problem, which is significantly more difficult than that of identifying the ingredients alone.

Volume Estimation. Estimating the total food amount can be addressed in the context of multi-view 3D reconstruction or single-view volume estimation. Multi-view reconstruction approaches [24], [25] largely rely on on traditional computer vision techniques. [26] estimated the contour of the food from three different views and matched it with a predefined library to estimate the volume. However, their library used only nine types of food with over-simplified shapes. Our work 
does not depend on any predefined library and only needs images and ingredient amounts. [27] used faster-RCNN to detect food and applied graph cuts to find the contour to estimate volume. However, food images in their dataset are well-balanced and easily segmentable. Compared with them, real-world datasets have a highly unbalanced distribution of ingredients, dominated by meal images with a complex mix of many ingredients.

In the significantly more challenging single view setting, [2] aimed to recognize the contents of a meal first then predict the calories for home-cooked foods. Their method is based on deep learning for classification and depth estimation to calculate the volume and calories. [28] used GANs, however their approach requires densely annotated datasets, which are not available for real-world data. The systems in [29] either uses size-known reference objects, including rice grains, or special devices, like inertial sensors and stereo cameras.

Estimating relative ingredient amounts, on the other hand, does not require reference objects or special devices. Methods estimating relative ingredient amounts can be applied to food images acquired in uncalibrated settings. Furthermore, while previous work calculated the amounts of the meal as a whole, estimating relative amounts of individual ingredients provides important additional information that can be linked to knowledge sources such as the micronutrition, wellness impact of individual food categories, etc. [30] was the first to attempt to predict relative ingredient amounts with CNNs. However, their methods were not significantly better than the retrieval baselines. In this work, we leverage retrievalinspired cross-modal image-text embeddings and minimize the Wasserstein distance of the food amount transport, driven by domain-specific ingredient subgroups. Experiments show that this approach can lead to new state-of-the-art on this challenging task.

\section{METHODS}

Ingredient relative amount prediction is a novel and ambiguous task. We first introduce some notations and define the problem in subsection III-A We start with the im2recipe retrieval system in subsection III-B. As the problem involves both ingredient detection and estimation, we detect the ingredients from embeddings as in subsection III-C and predict the amounts given the embeddings and the detected ingredients in subsection III-D, Figure 1 illustrates our approach.

\section{A. Notation and Problem Definition}

- Relative Amount. Given recipe $\mathbf{x}_{\mathbf{R}}$, we define the relative amount vector $\mathbf{v}=\left(v_{1}, \ldots, v_{i}, \ldots, v_{I}\right) \in \mathbb{R}_{>0}^{I}$, where $I$ is the total number of ingredients and $v_{i}=\frac{C}{M} m_{i}$ is the relative amount of the $i-t h$ ingredient, with $m_{i} \geq 0$ the absolute mass of ingredient $i$ in grams, $M=\sum_{i}^{I} m_{i}$ the total mass of all ingredients, and $C$ a normalizing constant. For convenience, we set $C=1000 \mathrm{gr}$. $v_{i}=0$ when the $i-t h$ ingredient is not present in the recipe.

- Ingredient Detection. Given recipe $\mathbf{x}_{\mathbf{R}}$, define the Ingredient Detection Vector $\mathbf{y}=\mathbf{1}_{\{\mathbf{v}>0\}} \in\{0,1\}^{I}$.
- Ingredient Distance. Let $\boldsymbol{M} \in[0,1]^{I \times I}$ be a matrix derived from substitution groups. $M_{i j}=M_{j i}, M_{i j} \geq$ $0, M_{i i}=0$. For ingredient triplet $(i, j, k)$, if ingredient $j$ is more similar to ingredient $i$ than ingredient $k$ determined by substitution groups, $M_{i j}<M_{i k}$. The matrix $M$ is called Ingredient Distance Matrix.

- Predicted quantities. We use the "hat" notation to refer to predicted quantities. For instance, $\hat{\mathbf{v}}=$ $\left(\hat{v}_{1}, \ldots, \hat{v}_{i}, \ldots, \hat{v}_{I}\right)$ refers to the amount vector predicted from an image.

\section{B. Retrieval Model}

The retrieval model is based on [6] that embeds a pair of text and image into a shared Food Space. The model is trained to maximize the similarity between a positive pair (e.g. text and image come from the same recipe), meanwhile minimizing that between a negative pair (e.g. text and image come from different recipes).

Formally, the retrieval model takes a pair of text (including title, ingredients and instructions) and image $\left(\mathbf{x}_{\mathbf{R}}, \mathbf{x}_{\mathbf{I}}\right)$ as input. Text $\mathbf{x}_{\mathbf{R}}$ is first trained with a Word2vec model that embeds each word into a vector. Title is treated as one sentence and is forwarded through an LSTM. The final hidden status is used as the feature of title. Ingredients go through a bidirectional LSTM to mitigate the influence of initial order as ingredients should be unordered. Instructions are more complicated because they usually contain several sentences that frequently exceed a hundred words. To effectively remember the longterm dependency in instructions, a two-layer LSTM structure is applied; the first layer is used to encode each step of the instructions into a vector. These "step vectors" are forwarded through another LSTM as a sequence and the final hidden state is used as the representation of the instructions. The features from title, ingredients and instructions are concatenated and passed through a fully-connected layer to yield the output $\mathbf{z}_{\mathbf{R}}$ of the Text Encoder,

$$
\mathbf{z}_{\mathbf{R}}=F_{t x t}\left(\mathbf{x}_{\mathbf{R}}\right) .
$$

Image is encoded by a ResNet50 [31]. The result after average pooling is used as the the initial feature and is passed through a fully-connected layer to get the output of IE. The output image feature $\mathbf{z}_{\mathbf{I}}$ has the same dimension as the text feature above. This process is encapsulated in the following model:

$$
\mathbf{z}_{\mathbf{I}}=F_{i m g}\left(\mathbf{x}_{\mathbf{I}}\right) .
$$

Based on the assumption that text features and image features share the Food Space, a fully-connected layer is applied to both text and image features. The mapping uses a weight sharing mechanism to further align the features from the two modalities in the same space:

$$
\begin{aligned}
\mathbf{q}_{\mathbf{R}} & =F_{f c}\left(\mathbf{z}_{\mathbf{R}}\right), \\
\mathbf{q}_{\mathbf{I}} & =F_{f c}\left(\mathbf{z}_{\mathbf{I}}\right),
\end{aligned}
$$

where $q_{\mathbf{R}}$ and $\mathbf{q}_{\mathbf{I}}$ are the final text feature and image feature in the Food Space. 
Loss is designed to minimize the similarity between a positive pair and maximize that between a negative pair. Cosine similarity is applied. We applied hard-sample mining [32] to make the model focus on the most difficult pairs. The loss $L_{r e t}$ in one batch is defined as

$$
\begin{aligned}
L_{r e t} & =\frac{1}{N} \sum_{i=1}^{N} \max \left(0, m-\cos \left(\mathbf{q}_{\mathbf{R}_{i}}, \mathbf{q}_{i}\right)+\cos \left(\mathbf{q}_{\mathbf{R}_{i}}, \mathbf{u}_{j}\right)\right) \\
i, j \in[1, N] \text { and } i \neq j &
\end{aligned}
$$

where $N$ is the batch size, $m$ is the margin between positive pair similarity and negative pair similarity ( $m$ is set to 0.3 by cross validation), $\cos \left(\mathbf{q}_{\mathbf{R}_{i}}, \mathbf{q}_{\mathbf{I}_{i}}\right)$ is the cosine similarity between a positive pair and $\cos \left(\mathbf{q}_{\mathbf{R}}, \mathbf{u}_{j}\right)$ is that between a negative pair. Hard-sample mining is applied here to choose the most difficult sample $\mathbf{u}_{j}$ (could be text or image), which is defined as the most similar negative sample with $\mathbf{q}_{\mathbf{R}}$.

\section{Ingredient Detection}

As the problem ingredient relative amount prediction involves both ingredient detection and estimation, after getting the image embedding $\mathbf{q}_{\mathrm{I}}$ from the retrieval system in subsection III-B, we first do ingredient detection using an ingredient detector, as in Figure 1. The soft probability prediction is

$$
\mathbf{p}=F_{i d}\left(\mathbf{q}_{\mathbf{I}}\right) .
$$

$p_{i} \in[0,1], i=1,2, \ldots, I$.

We minimize the positive sample weighted binary cross entropy loss between $\mathbf{p}_{\mathbf{x}}$ and $\mathbf{y}$. More specifically,

$$
L_{i n g r}=-\sum_{i=1}^{I}\left[w_{i} y_{i} \log p_{i}+\left(1-y_{i}\right) \log \left(1-p_{i}\right)\right]
$$

where $w_{i}$ is the positive sample weight, which is essential as ingredients are sparse.

To stabilize training, we set

$$
w_{i}=\min \left(t, \frac{\sum_{r \in R}\left(1-y_{r_{i}}\right)}{\sum_{r \in R} y_{r_{i}}}\right),
$$

where $R$ is the collection of recipes in the training set, $r$ is a recipe and $t$ is a threshold. In our experiment, $t$ is set to 4 by cross validation.

The hard prediction is the soft probability prediction thresholded to 0.5 ,

$$
\hat{\mathbf{y}}=\mathbf{1}_{\{\mathbf{p}>0.5\}} \text {. }
$$

$\hat{\mathbf{y}}$ is then passed to AP in Figure 1 to guide the amount prediction.

\section{Amount Prediction}

Given the image embedding $\mathbf{q}_{\mathbf{x}}$ from subsection III-B and ingredient detection vector $\hat{\mathbf{y}}$ from subsection III-C, we train an amount predictor, as in Figure 1. More specifically,

$$
\hat{\mathbf{v}}=F_{a p}\left(\mathbf{q}_{\mathbf{I}} \oplus \hat{\mathbf{y}}\right) \odot \hat{\mathbf{y}},
$$

where $\oplus$ means concatenation and $\odot$ denotes element-wise multiplication.
We minimize the Wasserstein distance [33] between $\hat{\mathbf{v}}$ and v. The loss function is

$$
L_{a m}=W_{p}^{p}(\hat{\mathbf{v}}, \mathbf{v}, \boldsymbol{M}),
$$

where $M$ is the ingredient distance matrix defined in subsection III-A Equation (11) can be minimized with the method in [33].

\section{EXPERIMENTS}

\section{A. Implementation Details}

The architectures of ID and AP are shown in Figure 1 Both are fully connected networks with leaky ReLU except the output layers. The negative slope of the leaky ReLU is 0.2. The output layer of ID is activated with sigmoid while the output layer of AP is activated with softmax.

The retrieval system, ID and AP are all optimized with Adam optimizer. The learning rate is $10^{-4}$ and the batch size is 64 . The three parts are learned sequentially in three incremental stages: 1) retrieval system, 2) ingredient detection, and 3) amount prediction. We find that fine tuning previous parts using weighted sum loss does not significantly improve performance. When training the retrieval system, we follow [3] and [6] where the ingredient amounts are not used. During test time, the image is first passed through IE to get an embedding representation $\mathbf{q}_{\mathbf{x}}$. Then $\mathbf{q}_{\mathbf{x}}$ is passed through ID to get $\hat{\mathbf{y}}$. Finally $\mathbf{q}_{\mathbf{x}}$ and $\hat{\mathbf{y}}$ are passed through AP to get the amounts.

\section{B. Dataset and Metrics}

Our dataset is based on Recipe1M [3] which includes over one million recipes scraped from the Internet including titles, ingredients descriptions with units and amounts ( 1 cup of flour) and instructions. About 400k of the recipes have images. For amount analysis, we use the 80k subset of Recipe1M annotated for amounts and with images in [30].

Ingredients. As the number of unique ingredients identified in Recipe $1 \mathrm{M}$ is over $16 \mathrm{k}$ including single and plural forms (egg, eggs) and nearly the same ingredient with different names (msg, accent_seasoning), we use the canonical ingredient construction in [30], which reduces the number of ingredients to $1.4 \mathrm{k}$ with $95 \%$ coverage for a more compact ingredient list. After removing the non-food ingredients like tin_foils and skewers, the number of ingredients is 1362. ID and AP are trained with the 48k training data of the annotated recipes while the retrieval system is trained with the training part of recipes with amounts and the rest of recipes with images that are not annotated for amounts, about $371 \mathrm{k}$ in total.

Ingredient Substitution Groups. The $1.4 \mathrm{k}$ ingredient list still contains ingredient substitutes like (butter, margarine) which should not be penalized as much as predicting butter as apples. Therefore, we construct ingredient substitution groups both for learning and evaluation. We first select ingredient pairs whose cosine similarity in Word2vec embedding space, which is trained on the instructions, ingredients and titles of Recipe1M, is greater than 0.6, resulting in five neighbors per ingredient on average. Human annotators then approve or 
reject the selected pairs. They can also add more pairs according to ingredient ontology, like (strawberries, mixed_fruits). 3142 out of 6342 ingredient pairs are accepted by human annotators. Next, we treat ingredients as nodes and approved or added pairs as edges of a graph. We compute the connected components of the graph and each connected component is one substitution group. This results in 172 substitution groups. An example of an ingredient group created is: duck fat, chicken fat, pork fat, bacon drippings and lard; that is, forms of animal fat. The ingredient distance matrix $M$ is then calculated as follows:

- Ingredient $i, j$ belong to the same substitution group: $M_{i j}=0$.

- $i, j$ belong to different substitution groups: $M_{i j}=$ $1-\cos \left(\mathbf{e m}_{\mathbf{i}}, \mathbf{e m}_{\mathbf{j}}\right)$, where $\cos (\cdot, \cdot)$ is cosine distance and $\mathbf{e m}_{\mathbf{i}}$ is the Word2vec embedding of ingredient $i$.

We report three evaluation metrics, the first two evaluate ingredient detection and the last evaluates amounts, all in the context of substitution groups. The number of common ingredients $c$ between $\mathbf{y}, \hat{\mathbf{y}}$ is

$c=\sum_{g_{i}} \min \left(\sum_{k \in g_{i}} y_{k}, \sum_{k \in g_{i}} \hat{y}_{k}\right)$ where $g_{i}$ is a substitution group and $k$ is an ingredient.

The metrics are:

- CVG: The coverage of ground truth ingredient, calculated by $\frac{c}{\sum_{i=1}^{I} y_{i}}$

- IOU: Intersection over the union of ground truth and detected, calculated by $\frac{c}{\sum_{i=1}^{I} y_{i}+\sum_{i=1}^{I} \hat{y}_{i}-c}$

- EMD: Earth mover's distance between ground truth and predicted amounts, using $M$ as the distance matrix.

We also report group level metrics. The distance between two substitution groups is the cosine distance between group centroids in Word2vec embedding space. Suppose the group level distance matrix is $\boldsymbol{M}_{\mathbf{1}}$. For substitution groups $g_{i}, g_{j}, \quad M_{1_{i j}}=1-\cos \left(\frac{1}{\left|g_{i}\right|} \sum_{k \in g_{i}} \mathbf{e m}_{\mathbf{k}}, \frac{1}{\left|g_{j}\right|} \sum_{k \in g_{j}} \mathbf{e m}_{\mathbf{k}}\right)$ where $\left|g_{i}\right|,\left|g_{j}\right|$ are the sizes of the substitution groups and $\mathrm{k}$ is an ingredient. The ingredient detection and amount vectors of the ground truth are collapsed according to groups. More specifically, suppose there are $g$ substitution groups. Define the group-ingredient matrix as $\boldsymbol{G} \in\{0,1\}^{g \times I}, G_{i k}=1$ if ingredient $k$ belongs to group $g_{i}$. The collapsed amount vectors are $\mathbf{v}_{\mathbf{1}}=\boldsymbol{G} \cdot \mathbf{v}, \hat{\mathbf{v}}_{\mathbf{1}}=\boldsymbol{G} \cdot \hat{\mathbf{v}}$ and the collapsed ingredient detection vectors are $\mathbf{y}_{\mathbf{1}}=\mathbf{1}_{\{\boldsymbol{G} \cdot \mathbf{y}>0\}}, \hat{\mathbf{y}}_{\mathbf{1}}=\mathbf{1}_{\{\boldsymbol{G} \cdot \hat{\mathbf{y}}>0\}}$. The group level metrics are:

- CVG (Group): Group-level coverage, calculated by

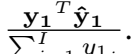

- IOU (Group): Group-level intersection over union, calculated by

$$
\frac{\mathbf{y}_{\mathbf{1}}^{T} \hat{\mathbf{y}}_{\mathbf{1}}}{\sum_{i=1}^{I} y_{1_{i}}+\sum_{i=1}^{I} \hat{y}_{1_{i}}-\mathbf{y}_{\mathbf{1}}^{T} \hat{\mathbf{y}}_{\mathbf{1}}} \text {. }
$$

- EMD (Group): Group-level earth mover's distance between $\mathbf{v}_{\mathbf{1}}$ and $\hat{\mathbf{v}}_{\mathbf{1}}$, using $\boldsymbol{M}_{\mathbf{1}}$ as the distance matrix.

Specifically, CVG (Group) and IOU (Group) can be viewed as calculated the usual way where substitutions are not considered and there are only 172 ingredients.

\section{Compared Methods}

We investigate both retrieval-based and prediction-based methods, as cross-modal retrieval can be seen as a baseline method to ingredient amount prediction by returning the ingredients and amounts of the retrieved recipe. Both ingredientlevel and group-level metrics are reported.

As for prediction-based methods, we study both ingredientlevel and group-level methods. Ingredient-level methods predict detailed amounts of all 1362 ingredients while group-level methods treat each substitution group as one ingredient. For ingredient-level methods, both ingredient-level and group-level metrics are reported. For group-level methods, only grouplevel metrics are reported.

The compared retrieval-based methods are:

- Retrieval: Use only the retrieval model in subsection III-B. Given an image, retrieve the top-1 recipe from a collection of 1000 randomly chosen recipes in the test set, following [3]. The ground truth recipe is not in the collection 2

- ATTEN[20]: The method in [20].

- ACME[21]: The method in [21].

We compare the retrieval model with [20] and [21] because their methods are the most similar to ours in terms of model architecture and loss function. A detailed comparison of the retrieval models is in Table I

For prediction-based methods, the compared ingredientlevel methods are:

- IE+AP: The dense method in [30]. The model uses a Resnet50 [31] to predict the ingredient amounts. The output layer is activated by softmax. The cross entropy between the ground truth and the predicted amounts is minimized. The result is thresholded to top-10 ingredients as the average number of ingredients in Recipe $1 \mathrm{M}$ is about ten [3].

- IE+RE+AP(Wass): The model only uses one network to predict the amounts from Food Space embeddings. Wasserstein distance between ground truth and predicted amounts using $M$ as the distance matrix is minimized. The result is thresholded to top-10 ingredients.

- IE+RE+AP(CE): The model architecture is the same as $\mathrm{IE}+\mathrm{RE}+\mathrm{AP}$ (Wass) but the loss function is cross-entropy as [30]. The result is thresholded to top-10 ingredients.

- IE+ID+AP: The model separates ingredient detection and amount prediction. IE is pretrained on UPMC [10] as in [30] and its weights are learned when training ID. AP is trained as in subsection III-D

- IE+RE+ID+AP(Wass): Our method in section III

- IE+RE+ID+AP(CE): The model architecture is the same as Figure 1 but AP does not use substitution group information or $M$ during training. The cross-entropy loss

${ }^{2}$ If the ground truth recipe is included in the collection, it will be the performance upper-bound as the model has access to the ground truth during test phase. The upper bound is: $\mathrm{CVG}=0.70, \mathrm{IOU}=0.59, \mathrm{EMD}=119.20$, $\mathrm{CVG}($ Group $)=0.71, \mathrm{IOU}($ Group $)=0.61, \mathrm{EMD}($ Group $)=237.90$. 
TABLE I: Retrieval-based Methods

\begin{tabular}{ccccc}
\hline & Title Encoder & Ingredient Encoder & Instruction Encoder & Loss Function \\
\hline Retrieval & LSTM & bidirectional LSTM & two-layer LSTM & hard mining triplet \\
ATTEN[20] & LSTM & bidirectional LSTM & two-layer LSTM & triplet \\
ACME[21] & - & bidirectional LSTM & skip-thought + LSTM & hard mining triplet \\
\hline
\end{tabular}

$\sum_{i=1}^{I} v_{i} \log \left(\hat{v}_{i}+\epsilon\right)$ between $\mathbf{v}, \hat{\mathbf{v}}$ is minimized. $\epsilon=10^{-6}$ is added for numerical stability as $\hat{\mathbf{v}}$ is sparse.

The group-level compared methods are:

- IE+RE+AP(Wass)(group): The network architecture is the same as IE+RE+AP(Wass) except that the output dimension of IE+RE+AP(Wass) is the number of ingredients 1362 while the output dimension of $\mathrm{IE}+\mathrm{RE}+\mathrm{AP}$ (Wass)(group) is the number of groups 172 . Wasserstein distance between the collapsed ground truth and predicted amounts using $\boldsymbol{M}_{1}$ as distance matrix is minimized. The result is thresholded to top-10 groups as the average number of groups in ground truth is also about ten. This is probably because ingredients within a substitution group do not frequently co-occur.

- IE+RE+ID+AP(Wass)(group): The group-level counterpart of IE+RE+ID+AP(Wass). ID is trained with substitution group-level binary cross-entropy loss. The positive sample weight is $\min \left(t, \frac{\sum_{r \in R}\left(1-y_{1_{r_{i}}}\right)}{\sum_{r \in R} y_{1_{r_{i}}}}\right)$ and $t$ is set to 4. For AP, Wasserstein distance between ground truth and predicted amounts using $\boldsymbol{M}_{1}$ as distance matrix is minimized.

\section{Results and Evaluation}

The performance of the ingredient-level methods and the group-level methods are shown in Table II The numbers are "mean over test set". The up arrows indicate the higher the better and the down arrows indicate the lower the better. The best method is marked bold.

Retrieval Methods Comparison. Comparing Retrieval, ATTEN [20] and ACME[21], our retrieval model achieves the best performance. Retrieval-based methods and predictionbased methods involving RE both use Food Space, but in different ways. Retrieval uses Food Space to find the nearest recipe neighbor, while prediction-based methods involving RE take Food Space features for further detection and estimation. Retrieval methods are all outperformed by IE+RE+ID+AP, which shows retrieval-based methods have their own limitations. For retrieval performance versus the number of recipes in the collection, please see our supplemental material.

Importance of RE (Food Space feature). Table II demonstrates RE (Food Space feature) is the most important component for both ingredient detection and amount prediction. This is because IE learns to extract image features highly relevant to the corresponding recipe, including title and instructions information when training the retrieval model. The Food Space features are then used to condition ID and AP and the performances are improved.

Importance of ID. Table II demonstrates ID improves CVG and IOU and only slightly degrades EMD. ID also provides information complementary to RE as IE+RE+ID+AP has the highest CVG and IOU and top-3 EMD.

Role of Substitution Groups during Training. $\mathrm{IE}+\mathrm{RE}+\mathrm{ID}+\mathrm{AP}(\mathrm{CE})$ and $\mathrm{IE}+\mathrm{RE}+\mathrm{ID}+\mathrm{AP}$ (Wass) have the same ID so they have the same CVG and IOU. IE+RE+ID+AP(Wass) utilizes substitution information when training AP but IE+RE+ID+AP(CE) does not. $\mathrm{IE}+\mathrm{RE}+\mathrm{ID}+\mathrm{AP}$ (Wass) has a slightly better EMD because the model learns to predict results with larger optimal flows for ingredient pairs with smaller distances. However, compare IE+RE+AP(Wass) with IE+RE+AP(CE), IE+RE+AP(Wass) utilizes substitution information during training while $\mathrm{IE}+\mathrm{RE}+\mathrm{AP}(\mathrm{CE})$ does not. The ingredient detection performance of IE+RE+AP(Wass) is severely degraded. This is because IE+RE+AP(Wass) tends to predict multiple ingredients from the same substitution group to match the amounts, see subsection IV-E which is not desired by users. Therefore, substitution information can improve AP but using it for ID still needs further research.

Ingredient-level vs Group-level Methods. Ingredientlevel methods predict detailed ingredient-level amounts while group-level methods view each group as a whole and can only predict group-level results. Table II shows that IOU (Group) is always higher than the ingredient level IOU as predicting multiple ingredients within the same group is penalized for ingredient-level metrics but not for group-level metrics. EMD (Group) is larger than ingredient level EMD because ingredient-level methods are trained to look for optimal transportations between close ingredient neighbors if the pair does not belong to the same group, which are much closer than cluster centroids used in EMD (Group). Therefore, the distances become larger. IE+RE+ID+AP(Wass)(group) performs the best if only group-level metrics are needed.

\section{E. Results on Sample Images}

Figure 2 highlights a cross-section of performance of the IE+RE+ID+AP(Wass) model. There, the top, middle and bottom rows, show cases of good, average and poor performance, respectively. Additionally, Figure 2 displays a comparison of prediction results from IE+RE+AP(Wass) and IE+RE+ID+AP(Wass) with ground truth amounts. Table III gives the specific performance values for both these methods on the examples shown on Figure 2. For optimal earth mover's distance flow visualization please see supplemental material.

In Cane Sauce (For Dippin' Chicken), there is only one match in IE+RE+AP(Wass), mayonnaise, on the other hand, every ingredient in ground truth is in IE+RE+ID+AP(Wass). However, as the major ingredient in ground truth is mayonnaise, IE+RE+AP(Wass) predicts multiple similar salad 
TABLE II: Performance of Compared Methods.

\begin{tabular}{ccccccc}
\hline Method & CVG $\uparrow$ & IOU $\uparrow$ & EMD $\downarrow$ & CVG (Group) & IOU (Group) & EMD (Group) \\
\hline Retrieval & 0.51 & 0.34 & 191.13 & 0.54 & 0.38 & 384.07 \\
ATTEN[20] & 0.47 & 0.32 & 205.19 & 0.50 & 0.35 & 407.73 \\
ACME[21] & 0.48 & 0.33 & 199.87 & 0.51 & 0.36 & 399.90 \\
IE+AP & 0.45 & 0.25 & 193.33 & 0.46 & 0.30 & 396.52 \\
IE+RE+AP(Wass) & 0.26 & 0.13 & $\mathbf{1 4 2 . 1 8}$ & 0.2 & 0.22 & 370.20 \\
IE+RE+AP(CE) & 0.50 & 0.28 & 145.30 & 0.51 & 0.35 & 307.78 \\
IE+ID+AP & 0.46 & 0.30 & 220.18 & 0.48 & 0.34 & 446.51 \\
IE+RE+ID+AP(CE) & $\mathbf{0 . 6 3}$ & $\mathbf{0 . 4 2}$ & 154.35 & 0.65 & $\mathbf{0 . 4 8}$ & 315.98 \\
IE+RE+ID+AP(Wass) & $\mathbf{0 . 6 3}$ & $\mathbf{0 . 4 2}$ & 147.29 & 0.65 & $\mathbf{0 . 4 8}$ & 319.63 \\
\hline IE+RE+AP(Wass)(group) & - & - & - & 0.63 & 0.36 & 293.54 \\
IE+RE+ID+AP(Wass)(group) & - & - & - & $\mathbf{0 . 7 5}$ & $\mathbf{0 . 4 8}$ & $\mathbf{2 9 1 . 1 6}$ \\
\hline
\end{tabular}

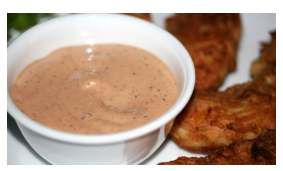

Cane Sauce (For Dippin' Chicken)

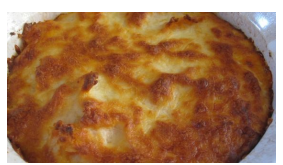

Creamy Baked Ziti

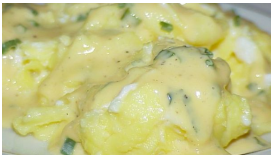

Scrambled Eggs
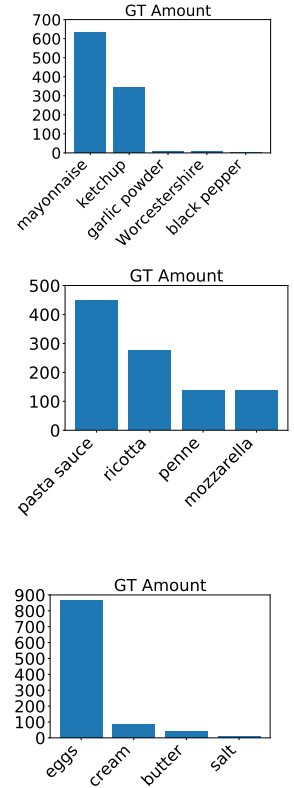
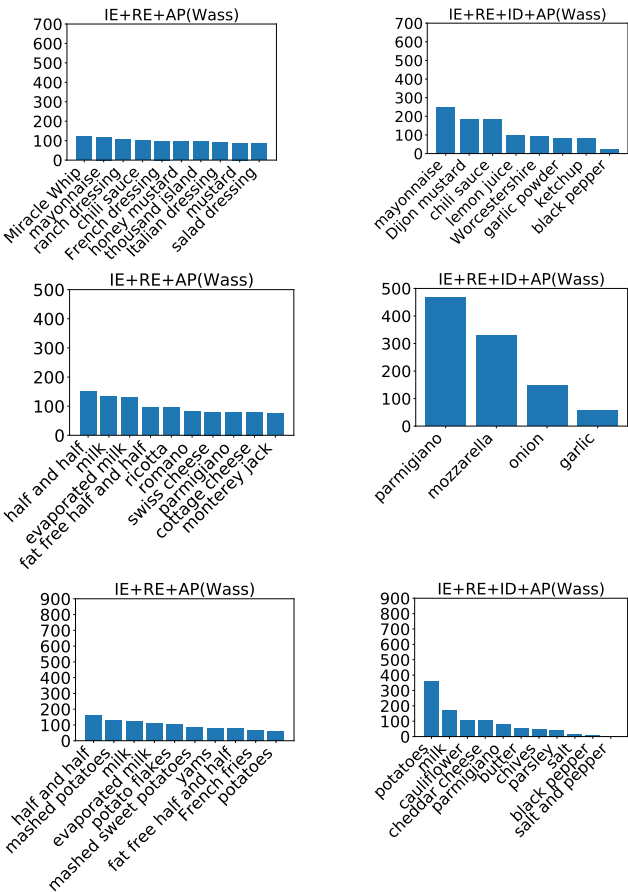

Fig. 2: The predictions of $\mathrm{IE}+\mathrm{RE}+\mathrm{AP}($ Wass), $\mathrm{IE}+\mathrm{RE}+\mathrm{ID}+\mathrm{AP}(\mathrm{Wass})$ and the ground truth amounts. Top row: IE+RE+ID+AP(Wass) performs much better than the average. Middle row: IE+RE+ID+AP(Wass) around the average. Bottom row: IE+RE+ID+AP(Wass) much worse than the average. The amounts are linearly scaled such that the total weight is 1000 grams.

TABLE III: Metrics of Examples in Figure 2

\begin{tabular}{llccc}
\hline Example & Method & CVG & IOU & EMD \\
\hline Cane Sauce (For & IE+RE+AP(Wass) & 0.20 & 0.07 & $\mathbf{4 0 . 0 6}$ \\
Dippin' Chicken) & IE+RE+ID+AP(Wass) & $\mathbf{1 . 0 0}$ & $\mathbf{0 . 6 3}$ & 63.77 \\
\hline Creamy Baked Ziti & IE+RE+AP(Wass) & $\mathbf{0 . 5 0}$ & 0.17 & 192.33 \\
& IE+RE+ID+AP(Wass) & $\mathbf{0 . 5 0}$ & $\mathbf{0 . 3 3}$ & $\mathbf{1 5 8 . 6 2}$ \\
\hline Scrambled Eggs & IE+RE+AP(Wass) & 0.00 & 0.00 & $\mathbf{2 5 2 . 9 1}$ \\
& IE+RE+ID+AP(Wass) & $\mathbf{0 . 5 0}$ & $\mathbf{0 . 1 5}$ & 347.04 \\
\hline
\end{tabular}

dressings, trying to match the total amount of mayonnaise, resulting in a smaller EMD. In Creamy Baked Ziti, the two cheeses in the ground truth are detected. IE+RE+AP(Wass) predicts more items in the cheese group, leading to a drop in IOU. As for amounts, the error in IE+RE+AP(Wass) mostly comes from milk and half-and-half, while the error in IE+RE+ID+AP(Wass) mostly comes from onion. In the Scrambled Eggs, none of the ingredients in ground truth is detected in IE+RE+AP(Wass) while butter and salt are detected in IE+RE+ID+AP(Wass). But for amounts, butter and salt take up less than $100 \mathrm{~g}$ in ground truth. For both methods, the amount error comes from matching other ingredients to eggs. IE+RE+ID+AP(Wass) has a larger error because of $100 \mathrm{~g}$ cauliflower, which has a larger difference to eggs than other ingredients predicted by the two methods. However, the scrambled eggs in the image look creamy, therefore, it might be reasonable to predict it as mashed potatoes, possibly with cauliflower. This highlights the methods' ability to produce plausible results even when presented with challenging images. 


\section{CONCLUSion}

We propose PITA, a deep learning architecture to predict the relative amount of each ingredient in a given food image. We first learn an image embedding representation from a cross-modal retrieval systems. Next, we detect ingredients from ingredient detection networks and predict amounts conditioned on ingredient detection results using an amount prediction network. Ingredient substitution groups are constructed to facilitate functional ingredient substitutions as a part of the evaluation metric and loss function. Experiments on annotated Recipe1M [3] show that our method generates state-of-the-art results, improving previous baselines. Even in the presence of challenging test examples, the model is still able to yield robust ingredient amount estimates.

\section{REFERENCES}

[1] O. Beijbom, N. Joshi, D. Morris, S. Saponas, and S. Khullar, "Menumatch: Restaurant-specific food logging from images," in IEEE Winter Conference on Applications of Computer Vision. IEEE, 2015, pp. 844851.

[2] A. Meyers, N. Johnston, V. Rathod, A. Korattikara, A. Gorban, N. Silberman, S. Guadarrama, G. Papandreou, J. Huang, and K. P. Murphy, "Im2calories: towards an automated mobile vision food diary," in Proceedings of the IEEE International Conference on Computer Vision, 2015, pp. 1233-1241.

[3] A. Salvador, N. Hynes, Y. Aytar, J. Marin, F. Ofli, I. Weber, and A. Torralba, "Learning cross-modal embeddings for cooking recipes and food images," in Proceedings of the IEEE conference on computer vision and pattern recognition, 2017, pp. 3020-3028.

[4] J. Chen and C.-W. Ngo, "Deep-based ingredient recognition for cooking recipe retrieval," in Proceedings of the 24th ACM international conference on Multimedia. ACM, 2016, pp. 32-41.

[5] F. J. He and G. A. MacGregor, "A comprehensive review on salt and health and current experience of worldwide salt reduction programmes," Journal of human hypertension, vol. 23, no. 6, p. 363, 2009.

[6] F. Han, R. Guerrero, and V. Pavlovic, "The art of food: Meal image synthesis from ingredients," arXiv preprint arXiv:1905.13149, 2019.

[7] T. Mikolov, I. Sutskever, K. Chen, G. S. Corrado, and J. Dean, "Distributed representations of words and phrases and their compositionality," in Advances in neural information processing systems, 2013, pp. 3111-3119.

[8] L. Bossard, M. Guillaumin, and L. Van Gool, "Food-101-mining discriminative components with random forests," in European Conference on Computer Vision. Springer, 2014, pp. 446-461.

[9] G. Ciocca, P. Napoletano, and R. Schettini, "Food recognition: A new dataset, experiments, and results." IEEE J. Biomedical and Health Informatics, vol. 21, no. 3, pp. 588-598, 2017.

[10] X. Wang, D. Kumar, N. Thome, M. Cord, and F. Precioso, "Recipe recognition with large multimodal food dataset," in 2015 IEEE International Conference on Multimedia and Expo (ICME). IEEE, 2015, pp. $1-6$.

[11] H. Kagaya, K. Aizawa, and M. Ogawa, "Food detection and recognition using convolutional neural network," in Proceedings of the 22nd ACM international conference on Multimedia, 2014, pp. 1085-1088.

[12] A. Singla, L. Yuan, and T. Ebrahimi, "Food/non-food image classification and food categorization using pre-trained googlenet model," in Proceedings of the 2nd International Workshop on Multimedia Assisted Dietary Management. ACM, 2016, pp. 3-11.

[13] M. Merler, H. Wu, R. Uceda-Sosa, Q.-B. Nguyen, and J. R. Smith, "Snap, eat, repeat: a food recognition engine for dietary logging," in Proceedings of the 2nd international workshop on multimedia assisted dietary management, 2016, pp. 31-40.

[14] X. Chen, Y. Zhu, H. Zhou, L. Diao, and D. Wang, "Chinesefoodnet: A large-scale image dataset for chinese food recognition," arXiv preprint arXiv:1705.02743, 2017.

[15] S. Mezgec and B. Koroušić Seljak, "Nutrinet: a deep learning food and drink image recognition system for dietary assessment," Nutrients, vol. 9, no. 7, p. 657, 2017.
[16] N. Martinel, G. L. Foresti, and C. Micheloni, "Wide-slice residual networks for food recognition," in 2018 IEEE Winter Conference on Applications of Computer Vision (WACV). IEEE, 2018, pp. 567-576.

[17] J. Qiu, F. P. W. Lo, Y. Sun, S. Wang, and B. Lo, "Mining discriminative food regions for accurate food recognition." in BMVC, 2019, p. 158.

[18] J. Chen, L. Pang, and C.-W. Ngo, "Cross-modal recipe retrieval: How to cook this dish?" in International Conference on Multimedia Modeling. Springer, 2017, pp. 588-600.

[19] M. Carvalho, R. Cadène, D. Picard, L. Soulier, N. Thome, and M. Cord, "Cross-modal retrieval in the cooking context: Learning semantic textimage embeddings," in The 41st International ACM SIGIR Conference on Research \& Development in Information Retrieval, 2018, pp. 35-44.

[20] J.-J. Chen, C.-W. Ngo, F.-L. Feng, and T.-S. Chua, "Deep understanding of cooking procedure for cross-modal recipe retrieval," in Proceedings of the 26th ACM international conference on Multimedia. ACM, 2018 , pp. 1020-1028.

[21] H. Wang, D. Sahoo, C. Liu, E.-p. Lim, and S. C. Hoi, "Learning crossmodal embeddings with adversarial networks for cooking recipes and food images," in Proceedings of the IEEE Conference on Computer Vision and Pattern Recognition, 2019, pp. 11 572-11 581.

[22] A. Salvador, M. Drozdzal, X. Giro-i Nieto, and A. Romero, "Inverse cooking: Recipe generation from food images," in Proceedings of the IEEE Conference on Computer Vision and Pattern Recognition, 2019 pp. 10453-10 462 .

[23] W. Min, L. Liu, Z. Luo, and S. Jiang, "Ingredient-guided cascaded multiattention network for food recognition," in Proceedings of the 27th ACM International Conference on Multimedia, 2019, pp. 1331-1339.

[24] A. Shashua and N. Navab, "Relative affine structure: Theory and application to $3 \mathrm{~d}$ reconstruction from perspective views," in Proceedings of the IEEE Conference on Computer Vision and Pattern Recognition, vol. 94, 1994, pp. 483-489.

[25] J. Dehais, M. Anthimopoulos, S. Shevchik, and S. Mougiakakou, "Twoview 3d reconstruction for food volume estimation," IEEE transactions on multimedia, vol. 19, no. 5, pp. 1090-1099, 2017.

[26] X. Zheng, Y. Gong, Q. Lei, R. Yao, and Q. Yin, "Multi-view model contour matching based food volume estimation," in International Conference on Applied Human Factors and Ergonomics. Springer, 2018, pp. 85-93.

[27] Y. Liang and J. Li, "Computer vision-based food calorie estimation: dataset, method, and experiment," arXiv preprint arXiv:1705.07632, 2017.

[28] S. Fang, Z. Shao, R. Mao, C. Fu, E. J. Delp, F. Zhu, D. A. Kerr, and C. J. Boushey, "Single-view food portion estimation: learning image-toenergy mappings using generative adversarial networks," in 2018 25th IEEE International Conference on Image Processing (ICIP). IEEE, 2018, pp. 251-255.

[29] T. Ege, Y. Ando, R. Tanno, W. Shimoda, and K. Yanai, "Image-based estimation of real food size for accurate food calorie estimation," in 2019 IEEE Conference on Multimedia Information Processing and Retrieval (MIPR). IEEE, 2019, pp. 274-279.

[30] J. Li, R. Guerrero, and V. Pavlovic, "Deep cooking: Predicting relative food ingredient amounts from images," in Proceedings of the 5th International Workshop on Multimedia Assisted Dietary Management. ACM, 2019, pp. 2-6.

[31] K. He, X. Zhang, S. Ren, and J. Sun, "Deep residual learning for image recognition," in Proceedings of the IEEE conference on computer vision and pattern recognition, 2016, pp. 770-778.

[32] A. Hermans, L. Beyer, and B. Leibe, "In defense of the triplet loss for person re-identification," arXiv preprint arXiv:1703.07737, 2017.

[33] C. Frogner, C. Zhang, H. Mobahi, M. Araya, and T. A. Poggio, "Learning with a wasserstein loss," in Advances in Neural Information Processing Systems, 2015, pp. 2053-2061. 